\title{
USO DE METODOLOGIAS ATIVAS NO ENSINO DE BALANÇO DE MASSA E ENERGIA: UM ESTUDO DE CASO EM TEMPOS DE PANDEMIA
}

\author{
DOI: 10.37702/2175-957X.COBENGE.2021.3522
}

Kátia Simone Teixeira da Silva de La Salles - katia.salles@ufma.br Universidade Federal do Maranhão AV. sao Luis rei de franca 48 65065-470 - sao luis - MA

Wendell Ferreira de La Salles - wendellsalles@hotmail.com Universidade Federal do Maranhão Avenida São Luís Rei de França 48 65065-470 - São Luís - MA

Resumo: Este trabalho relata a experiência da aplicação da aprendizagem baseada em projetos na disciplina de balanço de massa e energia ministrada aos alunos dos cursos de graduação em Química Industrial e Engenharia Química de uma Instituição de Ensino Superior do Estado do Maranhão. Trata-se de uma atividade construtivista, produção de mapa mental e vídeos, para substituir a tradicional resolução de lista de exercícios. O intuito da intervenção pedagógica foi de executar uma estratégia capaz de proporcionar certa flexibilidade no processo de ensino e aprendizagem na área tecnológica. Para seu desenvolvimento, a turma foi dividida em cinco grupos de quatro, o que propiciou a capacidade de interagir, compartilhar ideias, conhecimento e dúvidas, além de despertar a criatividade e a socialização. Como resultado, foi possível observar que a produção de mapa mental e dos vídeos se transfigurou num instrumento estimulante da aprendizagem, favorecendo a participação ativa dos estudantes na construção do seu conhecimento. A estratégia pedagógica aqui apresentada foi realizada durante o segundo semestre do ano de 2020, de forma remota, onde docentes e estudantes tiveram que se reinventar para aprender e ensinar, buscando novas metodologias e estratégias de ensino. A reação dos estudantes diante dessa estratégia, que foge ao ensino tradicional, foi extremamente positiva, demonstrando a importância do uso de diferentes formas de ensinar e aprender.

Palavras-chave: Mapa mental. Vídeos. Balanço de Massa e Energia. Metodologia 


\section{(C. COBENGE e IV Simpósio Internacional de Educação em Engenharia 2021 ie 28 a 30 de SETEMBRO

Baseada em Projeto 
USO DE METODOLOGIAS ATIVAS NO ENSINO DE BALANÇO DE MASSA E ENERGIA: UM ESTUDO DE CASO EM TEMPOS DE PANDEMIA

\section{INTRODUÇÃO}

Em função das restrições impostas devido à pandemia pela COVID-19, doença causada pelo novo coronavírus (Sars-Cov-2), as Instituições de Ensino tiveram seu fechamento temporário, obrigando-as, em um curto prazo de tempo, a trabalharem com o ensino remoto emergencial. O que era presencial passou a ser digital.

Neste contexto, professores tiveram que se reinventar em tempo recorde para aprender a usar ferramentais digitais e conseguir garantir, dentro das possibilidades, o aprendizado de seus alunos. A reflexão sobre a prática pedagógica se fez ainda mais necessária, e considerando Paulo Freire (1996, p. 63):

Não posso ser professor se não percebo cada vez melhor que, por não poder ser neutra, minha prática exige de mim uma definição. Uma tomada de posição. Decisão. Ruptura. Exige de mim que escolha entre isto ou aquilo.

Logo, foi preciso decidir e adotar, urgentemente, estratégias para fugir do tradicional ensino livresco e assim, garantir o processo de ensino e aprendizagem na nossa nova sala de aula: o ambiente virtual.

Mas, quais estratégias pedagógicas devem ser utilizadas para atrair os estudantes nesta nova sala de aula? Como promover a participação destes de forma mais ativa? E ainda, como motivá-los aumentando sua responsabilidade com a aprendizagem?

A busca por estratégias que cativem o interesse e motivação dos estudantes pela aprendizagem certamente é um desafio. Segundo Lima (2021 apud BROCHADO, 2020; OLIVEIRA, 2020), o cenário atual eleva o nível de dificuldade dos desafios, especialmente para os professores.

Os desafios são esperados e superá-los faz parte da capacidade de evoluir, aprender e se beneficiar dos saltos qualitativos que envolvem não apenas o produto, mas o processo (LIMA, 2017) o entremeio em que as mudanças acontecem. (LIMA, 2021)

Diante deste contexto, as metodologias ativas de aprendizagem aparecem com grande potencial para atender os desafios da educação atual.

A implementação dessas metodologias pode vir a favorecer uma motivação autônoma quando incluir o fortalecimento da percepção do aluno de ser origem da própria ação, ao serem apresentadas oportunidades de problematização de situações envolvidas na programação escolar, de escolha de aspectos dos conteúdos de estudo, de caminhos possíveis para o desenvolvimento de respostas ou soluções para os problemas que se apresentam alternativas criativas para a conclusão do estudo ou da pesquisa, entre outras possibilidades. Berbel (2011, p. 28).

Valente (2014) e Moran (2017, p.23) concordam com a reflexão de Berbel ao afirmarem que os estudantes assumem uma postura mais participativa ao trabalharem com 
metodologias ativas, criando oportunidades de construção do processo de aprendizagem, de forma flexível, interligada e híbrida. Nestes ambientes, o professor transcende a centralidade tradicional de transferidor de conhecimento, para aquele que propicia aos estudantes a serem protagonistas do seu percurso de aprendizagem.

O papel do educador não é o de encher o educando de conhecimento, de ordem técnica ou não, mas sim o de proporcionar, através da relação dialógica educadoreducando educando-educador, a organização de um pensamento correto em ambos (FREIRE, 2011, p.68).

Concluímos que são visíveis os benefícios pedagógicos do uso de metodologias ativas, cabendo ao professor verificar qual metodologia é o melhor recurso para o ensino da sua disciplina e quais circunstâncias irão proporcionar um aprendizado real aos seus estudantes. Neste processo, o professor, além de mediador, tem a função de motivador, despertando no estudante, a vontade de aprender (ÊLMOR FILHO et al., 2019).

Há uma série de estratégias ativas que podem ser utilizadas em sala de aula para auxiliar os estudantes ao longo do processo de aprendizagem, como: Instrução por pares (Peer Instruction); Sala de aula invertida (Flipped Classroom); Aprendizagem baseada em problemas (Problem Based Learning - PBL); Aprendizagem baseada em projetos (Project Based Learnin - PjBL) e Aprendizagem baseada em Jogos (Game Based Learning - GBL).

Dentre estas, a Aprendizagem Baseada em Projetos (PjBL) é considerada uma das práticas de ensino mais eficazes do século XXI (BENDER, 2014).

O Buck Institute for Education, define a metodologia PjBL da seguinte maneira:

A Aprendizagem Baseada em Projetos é um método de ensino pelo qual os alunos adquirem conhecimentos e habilidades trabalhando por um longo período de tempo para investigar e responder a uma questão, problema ou um desafio autêntico, envolvente e complexo.

Para Oliveira e Mattar (2018, p. 6) a metodologia PjBL "enfatiza as atividades realizadas por meio de projetos, cujo enfoque é a construção coletiva do conhecimento interdisciplinar na qual os alunos tornam-se protagonistas, ou seja, aprendem fazendo em cooperação com os colegas".

Com efeito, esse tipo de metodologia ativa é importante para que os estudantes sejam, de fato, o centro do processo de ensino aprendizagem.

Diante do exposto, e entendendo que essa abordagem pode viabilizar de maneira mais significativa o processo de ensino aprendizagem, será apresentada e discutida a estratégia pedagógica adotada na disciplina de Balanço de Massa e Energia Aplicada aos Processos Químicos de uma Instituição Federal de Ensino Superior do Estado do Maranhão. Trata-se de atividade construtivista, produção de mapas mentais e gravação de vídeos, para substituir a tradicional resolução de lista de exercícios.

O intuito deste relato é contribuir para modificar a prática pedagógica tradicional dos cursos superiores da área tecnológica, baseada apenas em aulas expositivas, possibilitando um ambiente sistemático e interativo para construção do conhecimento.

\section{INTERVENÇÃO PEDAGÓGICA}

O predomínio do ensino de técnicas de resolução de exercícios nas aulas de Balanço de Massa e Energia ainda é uma constante em muitas Universidades. Diante disso, buscamos, constantemente, novas metodologias de ensino que possam promover um 
maior interesse e envolvimento dos estudantes diante do conteúdo de estudo, e que, também, proporcionem uma mudança no modo de ensinar e aprender.

A prática pedagógica desenvolvida a partir de pressupostos teóricos de metodologia ativas foi aplica durante a disciplina de Balanço de Massa e Energia Aplicada a Processos Químicos composta por 20 acadêmicos dos cursos de bacharelado em Química Industrial e Engenharia Química de uma Instituição de Ensino Superior (IES) do Estado do Maranhão.

A aplicação envolvendo essa metodologia aconteceu durante as seis primeiras semanas do segundo semestre letivo do ano de 2020, onde foram trabalhados, seguindo 0 plano de ensino aprendizagem, os conteúdos de balanço de massa em processos químicos na ausência e presença de reações químicas, com correntes de reciclo e by pass.

O intuito da intervenção pedagógica foi de executar uma estratégia capaz de proporcionar certa flexibilidade no processo de ensino e aprendizagem na área tecnológica, onde uma parte do processo é planejada pelo professor (elaborar e disponibilizar materiais didáticos on-line, videoaulas, lista de exercícios) e a outra parte é conduzida pelos estudantes (exercitar o conteúdo proposto, esclarecer dúvidas).

A turma foi dividida em cinco grupos de quatro estudantes e a estratégia pedagógica utilizada foi organizada de acordo com o cronograma apresentado na Figura 1.

Figura 1: Estratégia Pedagógica

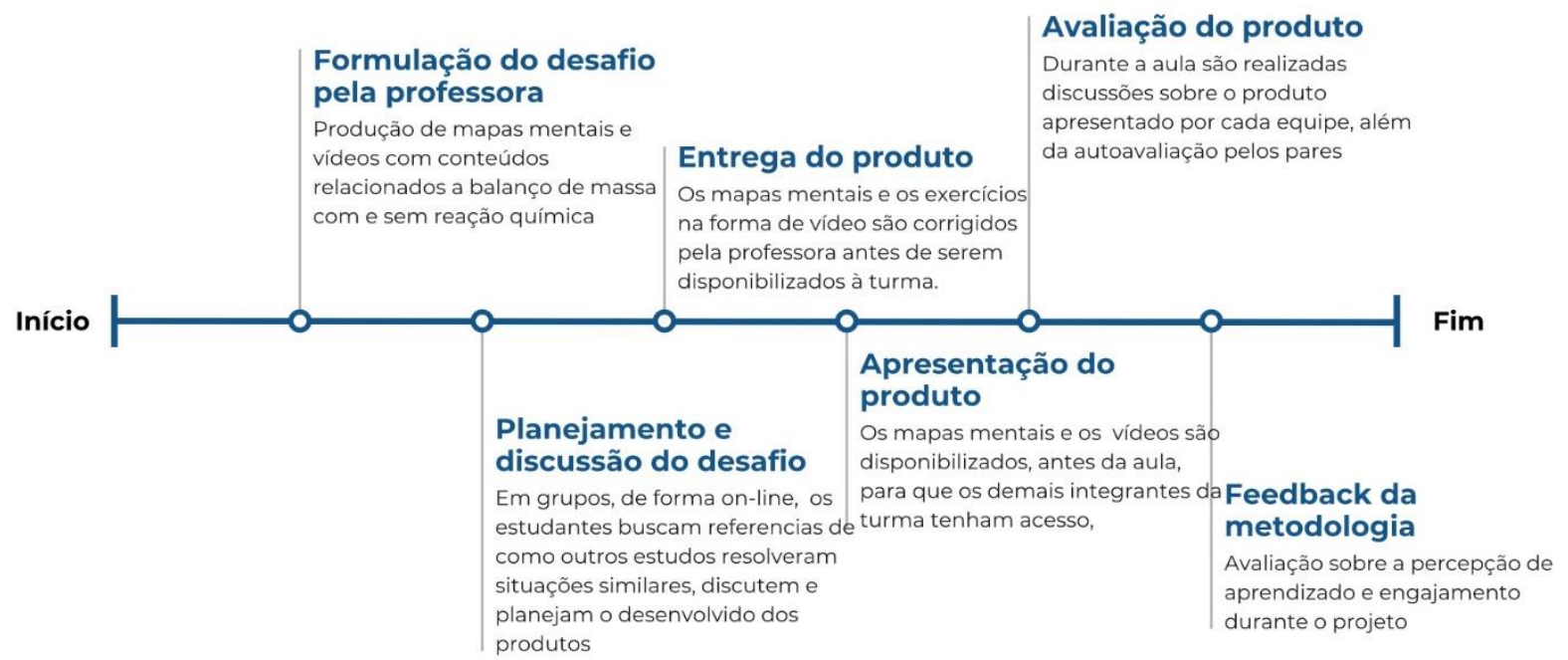

beautiful.ai

Fonte: Autor (2021)

Primeiramente, foi proposto aos estudantes que produzissem, individualmente, mapas mentais. O objetivo dessa atividade foi contribuir para o processo de aprendizagem auxiliando os alunos a memorizar os conteúdos teóricos abordados em sala de aula. 0 mapa mental ajuda os estudantes na compreensão de conteúdos mais densos e complexos, devido à possibilidade da criação e conexão de tópicos sobre temas específicos. Em seguida, os estudantes se reuniram, em grupo, de forma on-line, para produção de um novo mapa mental, este por sua vez, é basicamente o resultado da integração dos mapas individuas de todos os membros da equipe, onde os conceitos mais frequentes são colocados no novo mapa do grupo. 
A segunda etapa da atividade foi a resolução de problemas de processos químicos envolvendo reação química, com correntes de reciclo e/ou by pass, seguido de produção de vídeos como recurso didático. $\mathrm{O}$ uso de vídeos se destaca como um recurso didático complementar ao processo de ensino-aprendizagem possibilitando a construção do conhecimento de uma forma mais atrativa, criativa e significativa. A ideia central é a produção de vídeos dos problemas resolvidos, criando uma espécie de banco de vídeos com exercícios resolvidos para toda a turma.

Coube aos estudantes a escolha do ambiente e ferramentas para a elaboração dos produtos, os quais foram produzidos em plataformas diversas, a depender da equipe, e em seguida publicizados via sala on-line da disciplina no Google Classroom.

A Figura 2 mostra um exemplo de mapa mental produzido pelas equipes dos estudantes da disciplina de Balanço de Massa e Energia Aplicada aos Processos Químicos.

Figura 2: Exemplo de mapa mental produzido pela equipe $A$

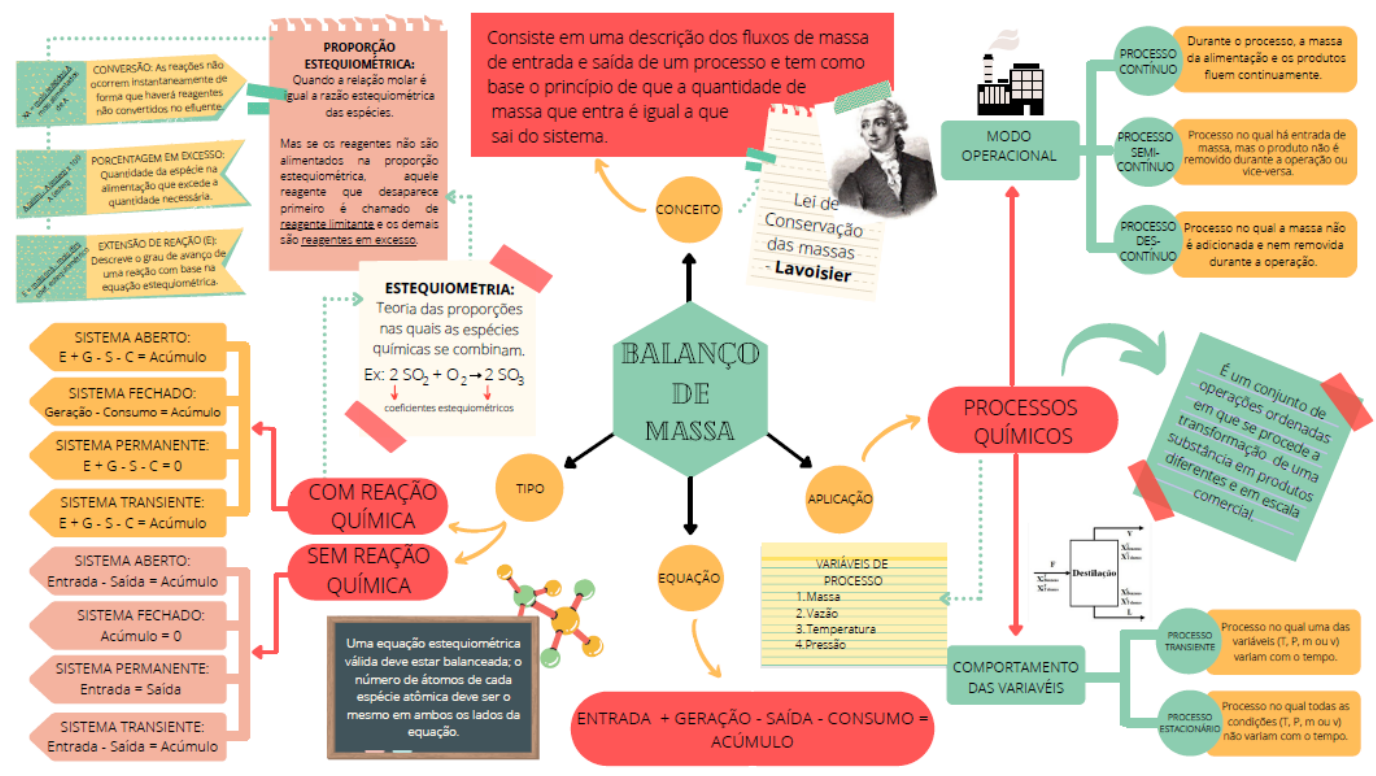

Fonte: Autor (2021)

A metodologia utilizada para desenvolver os produtos foi mesclada entre aulas síncronas, por meio da plataforma Google Meet, e momentos assíncronos (videoaulas e leitura material base disponível no ambiente virtual de aprendizagem da disciplina). Sendo assim, ao mesmo tempo em que o conteúdo da disciplina evolui, o estudante aplica e desenvolve o seu conhecimento na elaboração do mapa mental e vídeo, que evoluem em paralelo.

Com intuito de avaliar a utilização da metodologia PjBL, os estudantes foram convidados a preencher um questionário eletrônico ${ }^{1}$, de forma anônima, logo após a entrega dos produtos. O questionário foi desenvolvido na plataforma Google Forms, contemplando questões objetivas e subjetiva destinadas à avaliação da metodologia utilizada, e sua relação com os objetivos esperados: aprendizagem, motivação, engajamento e protagonismo.

\section{RESULTADOS E DISCUSSÕES}

${ }^{1}$ https://forms.gle/CAZb1Mep3ucPFz6HA 
Os resultados obtidos com base na resposta dos estudantes aos questionários aplicados na disciplina de Balanço de Massa e Energia aplicado aos Processos Químicos, sobre alguns aspectos qualitativos e quantitativos da atividade didática mapa mental e produção de vídeos, são apresentados nessa seção. O número de estudantes que responderam à pesquisa foi num total de 17, de uma turma composta por 20 estudantes.

Os resultados da avaliação são apresentados neste trabalho em forma de gráficos para uma melhor interpretação e visualização dos resultados das respostas coletadas no questionário.

A Figura 3 apresenta a avaliação geral dos estudantes quanto a utilização da metodologia PjBL na construção dos mapas mentais e vídeos.

Figura 3: Avaliação geral dos estudantes ao utilizarem a metodologia PjBL

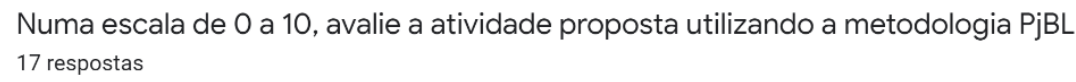

17 respostas

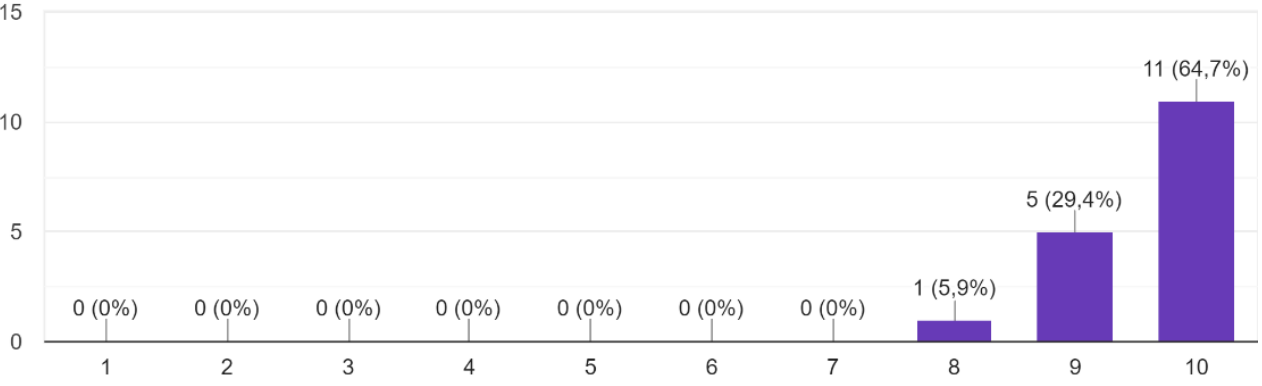

Fonte: Autor (2021)

Ao analisar os dados apresentados na Figura 3, verifica-se que as avaliações foram positivas quanto à utilização do método $\mathrm{PjBL}$ em Balanço de Massa e Energia. Este resultado representa, em termos gerais, o grau de aceitação da metodologia pelos estudantes durante a disciplina remota.

As Figuras 4 e 5 apresentam os resultados de percepção do processo de ensino e aprendizagem considerando a metodologia ativa utilizada.

Figura 4: Grau de confiança ao utilizar a metodologia PjBL

Quanto ao grau de confiança: Eu sinto confiança no que aprendi utilizando metodologias ativas 17 respostas

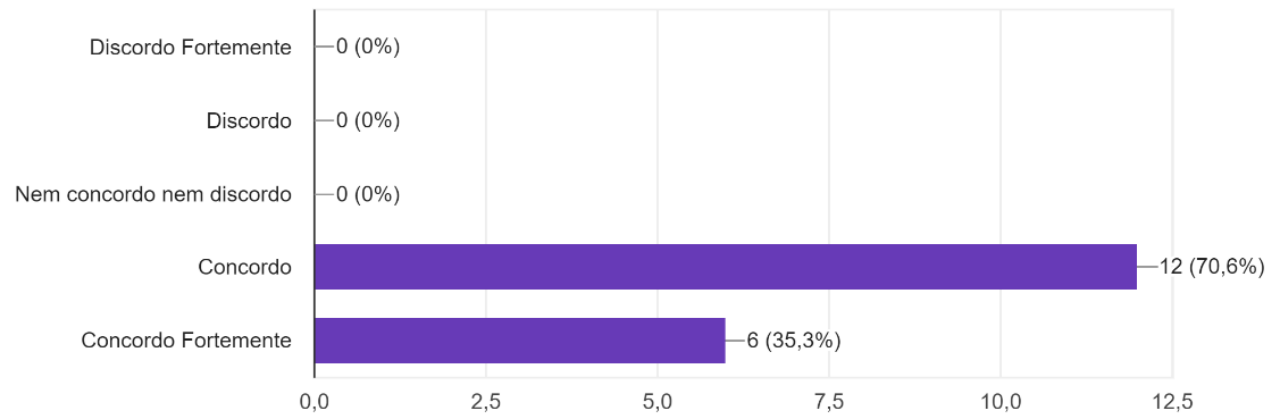


Fonte: Autor (2021)

Observa-se que $100 \%$ dos estudantes concordam que se sentiram confiantes no processo de ensino aprendizagem utilizando metodologias ativas e avaliam que a metodologia proporcionou oportunidades de buscar e usar informações em seu aprendizado (FIGURA 5).

Figura 5: Avaliação quanto a utilização de informações

A metododogia proporcionou oportunidades de buscar e usar informações em meu aprendizado

17 respostas

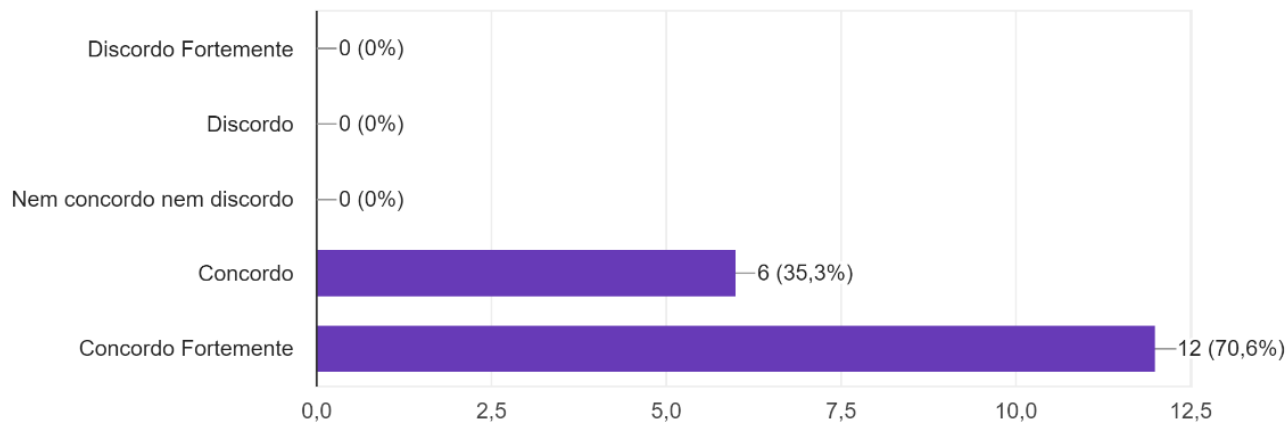

Fonte: Autor (2021)

De uma forma geral, de acordo com os resultados obtidos foi possível concluir que a metodologia utilizada, durante o desenvolvimento desta intervenção pedagógica, promoveu um bom índice no quesito de aprendizagem, atendendo às expectativas iniciais da professora.

Um outro aspecto investigado foi o trabalho em grupo, cujos resultados estão apresentados na Figura 6. 
Figura 6: Avaliação quanto ao trabalho em grupo (a) oportunidade de trabalhar em grupo; (b) dificuldade de se relacionar em grupo utilizando a metodologia PJBL

O método utilizado proporciona a oportunidade de trabalhar em grupo 17 respostas

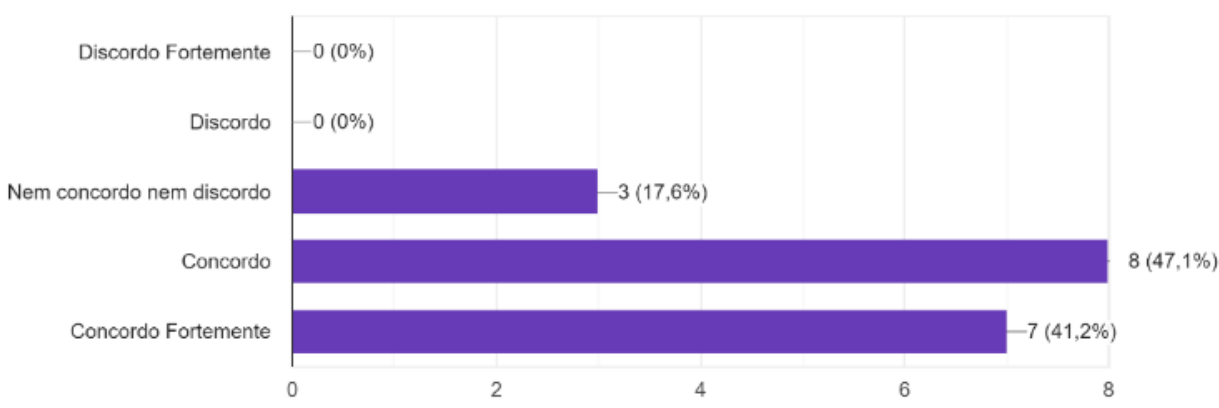

Sentiu dificuldade ao se relacionar com os outros integrantes utilizando a metodologi 17 respostas

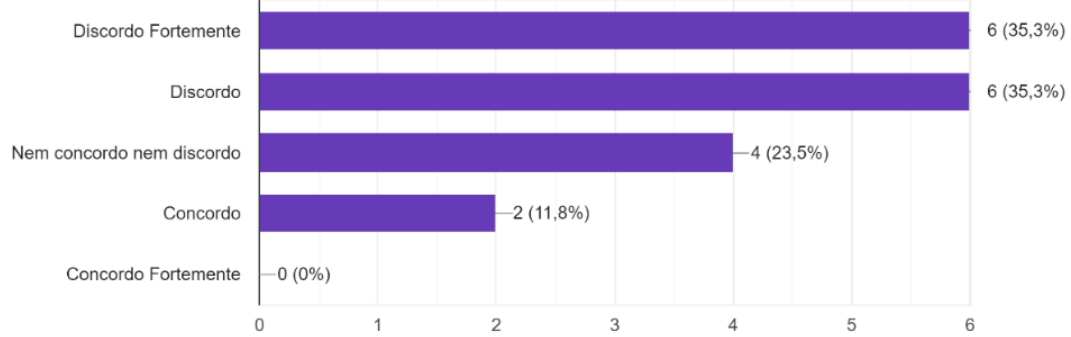

Fonte: Autor (2021)

Observa-se que a maioria dos estudantes, $88,3 \%$, afirmou que o método $\mathrm{PjBL}$ proporcionou a oportunidade de se trabalhar em grupo (FIGURA 6a), contudo 11,8\% sentiram dificuldades ao se relacionar com os demais integrantes da equipe (FIGURA 6b). Considerando que, em um contexto virtual, as mudanças na dinâmica das atividades presenciais para remotas modificam a concepção tradicional dos chamados trabalhos de/em grupo que passam a ser realizados por meio de ferramentas de comunicação online, é possível que esta avaliação esteja relacionada às dificuldades inerentes desta nova forma de execução de atividades em grupo.

A Figura 7 mostra a percepção dos estudantes quanto a utilização dessa metodologia em outras disciplinas. 
Figura 7: Percepção do uso de PjBL em outras disciplinas

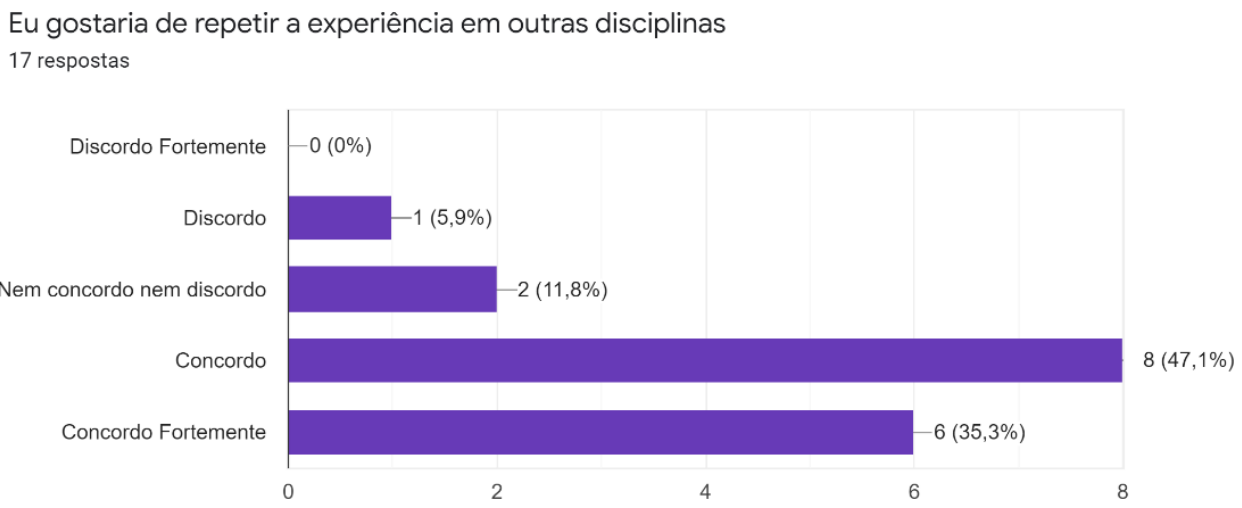

Fonte: Autor (2021)

Conclui-se que a experiência gerada a partir da prática da metodologia ativa de aprendizagem foi muito positiva e bem recebida pelos estudantes, considerando que a maioria, 82,4\%, gostariam de repetir a experiência quanto à utilização da metodologia $\mathrm{PjBL}$ em outras disciplinas (FIGURA 7).

Com relação a questão discursiva, a compilação dos dados do questionário mostrou os seguintes resultados:

a) O mapa foi uma atividade boa de ser feita além de prazerosa, bom para exercitar os conceitos e aprender mais a teoria, porém para o conteúdo abordado na segunda avaliação não foi possível exercitar tanto ajudaria a uma disciplina teórica, pois como exige bastante treino e exercícios práticos a segunda etapa o mapa não ajudou tanto. Mesmo assim serviu para reforçar a teoria e alguns conceitos importantes. (estudante 01);

b) Foi muito gostoso fazer esse trabalho, já é uma coisa que gosto de fazer. Amei muito!!! (estudante 02);

c) Preparo para prova - entendimento da matéria - ótima maneira para surgir e tirar dúvidas (estudante 03)

d) Método: trabalho em equipe 1. Melhor gestão do tempo pois é possível separar as responsabilidades. Exemplo: Todo mundo resolve uma alternativa da questão, uma pessoa faz o slide e outra pessoa grava o vídeo. 2. Cada um colabora com seus conhecimentos e habilidades relacionadas à disciplina. 3. O grupo possibilita que cada integrante consiga ampliar seus conhecimentos e aprimorar suas habilidades de interpretação e resolução de questões. (estudante 04);

e) Desenvolvimento mais rápido do exercício, trabalho em equipe, ajuda dos colegas. (estudante 05);

f) Bom método de aprendizagem visto que ao entender e explicar o conteúdo fixa melhor. Boa dinâmica em grupo onde requer trabalho em equipe. $\mathrm{E}$ o exercício proposto dentro dos padrões que foram feitos em sala, e uma boa revisão para prova (estudante 06);

g) Troca de ideias com colegas. Revisão de muitos assuntos que ajudam até total domínio no conteúdo. E melhoria pessoal na apresentação de trabalhos em frente às câmeras (estudante 07). 
A análise das respostas dos estudantes possibilitou considerar positiva a aplicação da metodologia ativa no processo de ensino aprendizagem na área tecnológica, especificamente nos conteúdos de Balanço de Massa e Energia Aplicado aos Processos Químicos.

Entendemos que o objetivo da intervenção pedagógica foi atingido, exemplificada pela seguinte resposta de um dos estudantes ao questionário: "Bom método de aprendizagem visto que ao entender e explicar o conteúdo fixa melhor. Boa dinâmica em grupo onde requer trabalho em equipe".

\section{CONSIDERAÇÕES FINAIS}

Neste trabalho foram apresentados considerações e resultados sobre a utilização da metodologia de aprendizagem baseada em projetos, obtendo como produto mapas mentais e vídeos educacionais, no contexto da disciplina Balanço de Massa e Energia aplicado aos Processos Químicos Industriais de uma IES Federal do Estado do Maranhão.

A experiência em adotar a metodologia $\mathrm{PjBL}$ com objetivo de produção de mapas mentais e vídeos foi uma novidade para a maioria dos estudantes da turma, pois estavam adaptados ao método tradicional de ensino: aulas expositivas, resolução de lista de exercícios, provas escritas, seminários.

Considerando os resultados do presente estudo, esses sugerem que os estudantes estão abertos ao uso de novas tecnologias para aprendizagem. De uma forma geral, a reação dos estudantes diante dessa estratégia, que foge ao tradicional ensino livresco, foi extremamente positiva, demonstrando a importância do uso de diferentes formas de ensinar e aprender na área tecnológica.

\section{REFERÊNCIAS}

BENDER, Willian N. Aprendizagem baseada em projetos: educação diferenciada para o século XXI. 1.ed. Porto Alegre: Penso, 2014.

BERBEL, Neusi Aparecida Navas. As metodologias ativas e a promoção da autonomia de estudantes. Semina: Ciências Sociais e Humanas, Londrina, v. 32, n. 1, p. 25-40, 2011.

Buck Institute for Education. What is Project Based Learning (PBL)? Disponível em: https://www.pblworks.org/what-is-pbl. Acesso em 04 abril 2021.

ELMÔR FILHO, Gabriel et al. Uma Nova Sala de Aula é Possível - Aprendizagem Ativa na Educação em Engenharia. 1. ed. Rio de Janeiro: LTC, 2019.

FREIRE, Paulo. Extensão ou comunicação? 15. Ed. São Paulo: Paz e Terra, 2011.

FREIRE, Paulo. Pedagogia da Autonomia: saberes necessários à prática educativa. São Paulo: Paz e Terra, 1996.

LIMA, Fernando Silvério. Quando as coisas acontecem antes de acontecer: educação em um cenário complicado. In: Janete Palú, Jenerton Arlan Schütz, Leandro Mayer (Org.). Desafios da educação em tempos de pandemia. Cruz Alta: Ilustração, 2020, p 259-273. 
MORAN, José. Metodologias ativas e modelos híbridos na educação. In: YAEGASHI, S. et al (Org.). Novas tecnologias digitais: Reflexões sobre mediação, aprendizagem e desenvolvimento. 1. Ed. Curitiba: CRV, 2017, p.23-35.

OLIVEIRA, N. A. A., MATTAR, J. Folhetim Lorenianas: aprendizagem baseada em projetos, pesquisa e inovação responsáveis na educação. Revista e-Curriculum, São Paulo. 1-23. v. 16, n.2, p. 341-363, 2018.

VALENTE, J. A. Blended learning e as mudanças no ensino superior: a proposta da sala... Educar em Revista, Curitiba, Brasil, Edição Especial n. 4/2014, p. 79-97. Editora UFPR.

\title{
USE OF ACTIVE LEARNING METHODOLOGIES IN THE TEACHING OF MASS AND ENERGY BALANCE: A CASE STUDY IN PANDEMIC TIMES
}

\begin{abstract}
This paper reports the experience of applying project-based learning in the mass and energy balance discipline taught to students of the undergraduate courses in Industrial Chemistry and Chemical Engineering of a Higher Education Institution in the State of Maranhão. It is a constructivist activity, producing mind maps and videos, to replace the traditional solution of problems. The purpose of this pedagogical intervention was to execute a strategy capable of providing some flexibility in the teaching and learning process in the technological area. For its development, the class was divided into five groups of four members, which provided the ability to interact, share ideas, knowledge and doubts, in addition to awakening creativity and socialization. As a result, it was possible to observe that the production of mind maps and videos became a stimulating tool for learning, encouraging the active participation of students in building their knowledge. The pedagogical strategy presented here was carried out during the second half of 2020 , remotely, where teachers and students had to reinvent themselves to learn and teach, seeking new teaching methodologies and strategies. The students' reaction to this strategy, which differs from traditional teaching, was extremely positive, demonstrating the importance of using different ways of teaching and learning.
\end{abstract}

Keywords: mind maps, videos, mass and energy balance, project-based learning 